\title{
Isochoric thermal conductivity of solid $n$-alkanes:
} hexane $\mathrm{C}_{6} \mathrm{H}_{14}$

\author{
V.A. Konstantinov, V.P. Revyakin, and V.V. Sagan \\ B. Verkin Institute for Low Temperature Physics and Engineering of the National Academy of Sciences of Ukraine \\ 47 Lenin Ave., Kharkov 61103, Ukraine \\ E-mail:konstantinov@ilt.kharkov.ua
}

Received December 10, 2010

\begin{abstract}
The isochoric thermal conductivity of solid $n$-hexane $\mathrm{C}_{6} \mathrm{H}_{14}$ has been investigated on three samples of different density in the temperature interval from $100 \mathrm{~K}$ to the onset of melting. In all the cases the isochoric thermal conductivity varied following a dependence which is weaker than $\Lambda \propto 1 / T$. The results obtained are compared with the thermal conductivities of other representatives of $n$-alkanes. The contributions of low-frequency phonons and "diffuse modes" to the thermal conductivity are calculated.
\end{abstract}

PACS: 66.70.-f Nonelectronic thermal conduction and heat-pulse propagation in solids; thermal waves, Keywords: $n$-alkanes, hexane, isochoric thermal conductivity, phonons, "diffusive" modes.

\section{Introduction}

Normal alkanes (n-paraffins) of the $\mathrm{C}_{n} \mathrm{H}_{2 n+2}$ type form a class of substances that are intermediate in changing over to long-chain polymers. $N$-alkanes have a comparatively simple structure and a molecular packing: in the solid state the axes of all molecules are always parallel to one another irrespective of a particular crystalline modification [1]. Owing to their relative simplicity, normal alkanes are naturally considered as the starting point for understanding the structural and termophysical properties of more complex long-chain compounds.

$\mathrm{N}$-alkanes exhibit an extremely diverse dynamic behavior both in the solid and liquid states. The melting temperature increases in this series of compounds with the length of the chain and its behavior is nonmonotonic: the $n$-alkanes with an add number of carbon atoms (odd $n$-alkanes) melt at comparatively lower temperatures than those with an even number of $\mathrm{C}$ atoms (even $n$-alkanes). An interesting effect is observed when the orthorhombic, monoclinic and triclinic structures alternate with the even and odd members of the series [1-3]. The even $n$-alkanes with $n=6-24(n=$ the number of $\mathrm{C}$ atoms) crystallize at low temperatures forming a triclinic cell. Heptane $(n=7)$ and nonane $(n=9)$ have an orthorhombic structure at low temperatures. Glass-like "rotational" phases with hexagonal symmetry were found in rather narrow temperature interval below the melting points of odd $n$-alkanes starting with $n=9$. The region of existence of the "rotational" phase increases with the length of the chain. Hexagonal modifications also occur in even $n$-alkanes starting with $n=22[1-4]$.
Short-chain $(n \leq 6)$ and "even" $n$-alkanes are the least studied members of the series. Previously, thermal conductivity was investigated only in "odd" $n$-alkanes with $n=9-19$ at constant pressure $30 \mathrm{MPa}$ [5]. We have investigated the isochoric thermal conductivity of methane [6], ethane [7] and propane [8]. Here we report the isochoric thermal conductivity of solid $n$-hexane $\left(\mathrm{C}_{6} \mathrm{H}_{14}\right)$ measured on samples of various densities in the temperature interval from $100 \mathrm{~K}$ to the onset of melting.

According to colorimetric data, $n$-hexane has only one crystallographic modification and melts at $T_{m}=177.8 \mathrm{~K}$ with the entropy of melting $\Delta S_{f} / R=8.85$ [9]. The crystal structure of $n$-hexane has been determined by the x-ray method at 90 and $158 \mathrm{~K}[2,10]$. It is triclinic, space group $P \overline{1}$, with one molecule in the unit cell. On elastic neutron scattering [11], the lattice mode peaks occur at $35,55,71$ and $93 \mathrm{~cm}^{-1}$, which agree well with the Raman scattering data [12] $\left(53,74\right.$ and $\left.87 \mathrm{~cm}^{-1}\right)$. It is found that in $n$-hexane the lowest intramolecular modes are separated from the lattice modes not so distinctly as in light $n$-alkanes. The Debye temperature may be evaluated as the upper boundary of the lattice modes $(\sim 102 \mathrm{~K})$.

\section{Experimental}

For a correct comparison with theory, the measurement must be made at a constant density of the sample to exclude the thermal expansion effect. Constant-volume investigations are possible for molecular solids having comparatively high compressibility coefficients. Using a high pressure cell, it is possible to grow samples of sufficient density which can be cooled then with practically invaria- 
ble volume whereas the pressure in the cell decreases. As samples of moderate densities are cooled the pressure in the sell drops to zero at a certain characteristic temperature $T_{0}$ and the isochoric condition is then broken. At and below $T_{0}$ isochoric data turn to isobaric at zero pressure. On further cooling, the sample can separate from the walls of the cell or its continuity can be disturbed. In constantvolume experiments melting occur in a certain temperature interval, and its onset shifts towards higher temperatures as the density of the samples increases. These measurements were made by a steady - state flow heat method in a coaxial geometry setup [13]. The samples were grown under different pressures (40-120 MPa), the temperature gradient over the measuring cell being about $1.5 \mathrm{~K} / \mathrm{cm}$. As the growth was completed, the capillary was blocked by cooling it with liquid nitrogen, and the samples were annealed below melting temperatures for about two hours to remove the density gradients. After the measurements the samples were evaporated into a thin-wall vessel, and the samples masses were determined through weighting. The molar volumes of the samples were found from known volume of the measuring cell and the sample mass. Purity of the $\mathrm{C}_{6} \mathrm{H}_{14}$ used was no worse than $99.8 \%$.

\section{Results and discussion}

The experimental results are shown in Fig. 1, which carries the smoothed thermal conductivity of the solid phase (solid lines) and the isobaric $(P=0, \mathrm{MPa})$ thermal conductivity (dashed line). The thermal conductivities of the samples grown under identical conditions (grown time, pressure and temperature gradient over the measuring cell) coincident within the experimental error, which is evidence of the fine - disperse state of the samples (considerable anisotropy of the thermal conductivity can be expected along and across the plane of the layers). The molar volumes $V_{m}$ of the samples, the temperatures $T_{0}$ of the onset

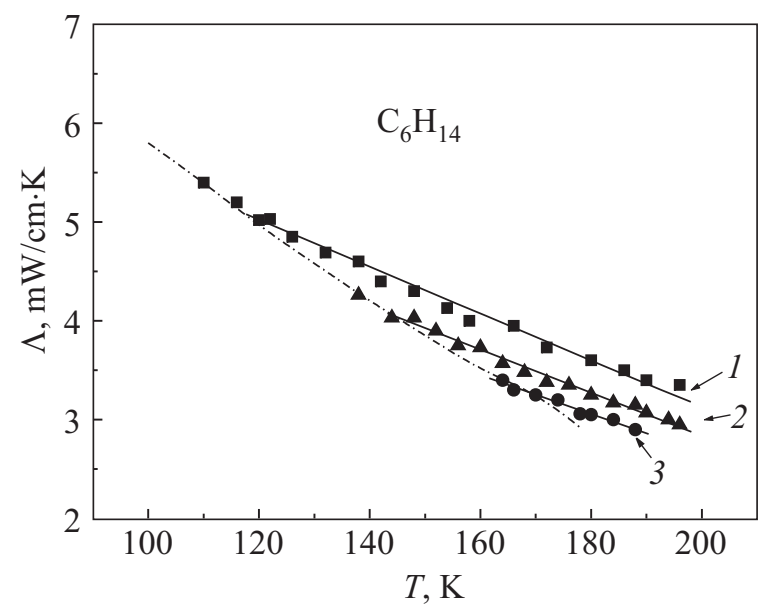

Fig. 1. The isochoric thermal conductivity of three solid $n$-hexane samples of various densities: №1(⿻), №2 ( $\mathbf{\Delta})$ и №3 (•). Solid lines are smoothed values of isochoric thermal conductivity. Dashed line is the thermal conductivity under atmospheric (zero) pressure. of experimental condition $V=$ const, and the temperatures $T_{m}$ of the onset of melting are given in Table 1. The Bridgman coefficient $g=-(\partial \ln \Lambda / \partial \ln V)_{T}$ was calculated from experimental data to be $7.6 \pm 0.6$ at $T=178 \mathrm{~K}$.

Table 1. Molar volumes $V_{m}$ of samples, temperatures $T_{0}$ of the onset of experimental condition $V=$ const, and temperatures $T_{m}$ of the onset of melting.

\begin{tabular}{c|c|c|c}
\hline \hline Number of sample & $V_{m}, \mathrm{~cm}^{3} /$ mole & $T_{0}, \mathrm{~K}$ & $T_{m}, \mathrm{~K}$ \\
\hline \hline 1 & 95.6 & 116 & 235 \\
2 & 96.7 & 142 & 210 \\
3 & 97.3 & 166 & 192 \\
\hline \hline
\end{tabular}

The isochoric thermal conductivity of all solid $n$-hexane samples decreases with increasing temperature following a dependences weaker than $\Lambda \propto 1 / T$. A similar behavior was observed previously in the low-temperature phase of ethane [7] and in propane [8]. It was noted previously that the thermal conductivity of long-chain odd $n$-alkanes $\left(\mathrm{C}_{9} \mathrm{H}_{20}-\mathrm{C}_{19} \mathrm{H}_{40}\right)$ has some features in common [5]. As the "rotational" phase melts, the thermal conductivity changes by about $35 \%$ and is independent of the chain length. The jump of the thermal conductivity on changing to the low temperature ordered phase decreases with the increasing length of the chain and makes $\sim 85 \%$ for $n$-undecane and $\sim 40 \%$ for $n$-nonadecane. The absolute value of thermal conductivity increases in the "rotational" phase with the increasing chain length.

The isobaric thermal conductivity of some $n$-alkanes is shown in Fig. 2 (data of this study and [5,7,8] along with the thermal conductivities of the liquid phases of these compounds measured immediately after melting $[5,14]$. Data for ethane, propane and hexane correspond to zero pressure; thermal conductivity of "odd" $n$-alkanes with $n=9-19$ was studied at constant pressure $30 \mathrm{MPa}$. Some information about structure and thermo physical properties of $n$-alkanes discussed is also available in Table 2. As noted above, the

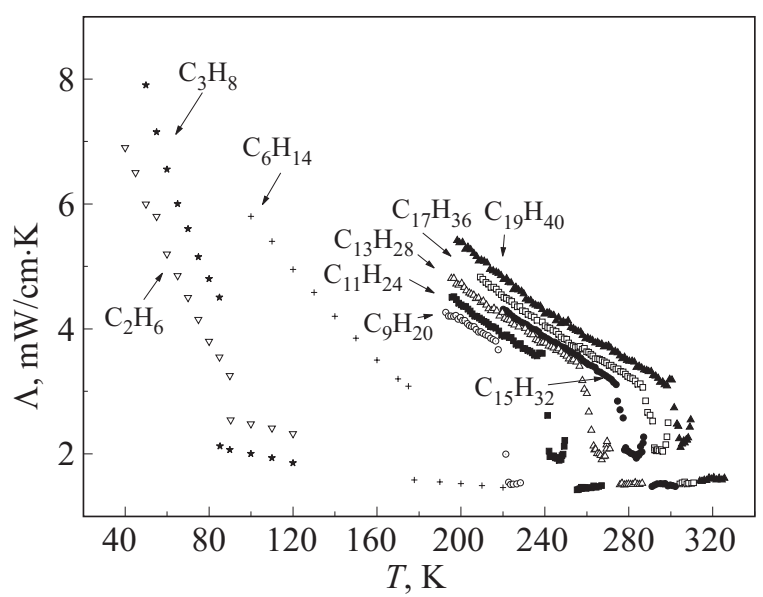

Fig. 2. Isobaric thermal conductivity of some n-alkanes (this study and $[5,7,8])$. Data for ethane, propane and hexane correspond to zero pressure; thermal conductivity of "odd" $n$-alkanes with $n=9-19$ correspond to pressure $30 \mathrm{MPa}$. 
isobaric thermal conductivity exhibits closely similar behavior in short and long-chain $n$-alkanes. On the transition from the ordered phase to a liquid the thermal conductivity of the $n$-alkanes starting with propane changes nearly twice and is independent of the total entropy of transitions and the chain length. Such change is much smaller in the case of spherical and elliptic molecules: for example, $\Delta \Lambda / \Lambda_{L}$ is only $20-30 \%$ in methane and ethane. This can be related to the higher degree of orientational ordering in solid long-chain $n$ alkanes as compared to spherical molecules.

Table 2. The structure of $n$-alkanes [2,5,10], the temperature $T_{\alpha-\beta}$ and the entropy $\Delta S_{\alpha-\beta} / R$ of the transition to the "rotational" phase, the temperature $T_{m}$ and the melting entropy $\Delta S_{m} / R$ [NIST Standard Reference Data: http://webbook.nist.gov/chemistry/form-ser.html], a complete change of the entropy and variations of thermal conductivity $\Lambda_{\alpha} / \Lambda_{L}$ during the ordered phase - liquid transition $[5,7,8,14]$.

\begin{tabular}{c|c|c|c|c|c|c|c}
\hline \hline Substance & Structure & $T_{\alpha-\beta}, \mathrm{K}$ & $\Delta S_{\alpha-\beta} / R$ & $T_{m}, \mathrm{~K}$ & $\Delta S_{m} / R$ & $\Delta S_{\alpha-L} / R$ & $\Lambda_{\alpha} / \Lambda_{L}$ \\
\hline \hline $\mathrm{C}_{2} \mathrm{H}_{6}$ & $P 2_{1} / \mathrm{n}, \mathrm{z}=2$ & 89.8 & 2.74 & 90.3 & 0.77 & 3.6 & 1.3 \\
$\mathrm{C}_{3} \mathrm{H}_{8}$ & $P 2_{1} / \mathrm{n}, \mathrm{z}=4$ & - & - & 85.5 & 4.95 & 4.95 & 2.2 \\
$\mathrm{C}_{6} \mathrm{H}_{14}$ & $P_{\overline{1}}, \mathrm{z}=1$ & - & - & 177.8 & 8.85 & 8.85 & 1.9 \\
$\mathrm{C}_{9} \mathrm{H}_{20}$ & $P_{\overline{1}, \mathrm{z}=1}$ & 217.2 & 3.48 & 219.7 & 8.47 & 12.0 & 2.4 \\
$\mathrm{C}_{11} \mathrm{H}_{24}$ & $P_{\text {bcn }}, \mathrm{z}=4$ & 236.6 & 2.9 & 247.6 & 10.8 & 13.7 & 2.4 \\
$\mathrm{C}_{13} \mathrm{H}_{28}$ & $P_{\text {bcn }}, \mathrm{z}=4$ & 255.0 & 3.6 & 267.8 & 12.8 & 16.4 & 2.3 \\
$\mathrm{C}_{15} \mathrm{H}_{32}$ & $P_{\text {bcn }}, \mathrm{z}=4$ & 270.9 & 4.1 & 283.1 & 14.7 & 18.8 & 2.1 \\
$\mathrm{C}_{17} \mathrm{H}_{36}$ & $P_{\text {bcn }}, \mathrm{z}=4$ & 284.3 & 4.8 & 295.1 & 16.4 & 21.2 & 2.0 \\
$\mathrm{C}_{19} \mathrm{H}_{40}$ & $P_{\text {bcn }}, \mathrm{z}=4$ & 296.0 & 5.6 & 304.0 & 18.8 & 24.3 & 2.0 \\
\hline \hline
\end{tabular}

The deviation of the isochoric thermal conductivity from the dependence $\Lambda \propto 1 / T$ in the orientationally-ordered phases of molecular crystals can be explained proceeding from the concept of the "lower limit to thermal conductivity" $[15,16]$. In this case the thermal conductivity can be calculated within the model in which heat is transported by both low-frequency phonons and "diffuse" modes. In simple Debye model the thermal conductivity can be described by the following expression

$$
\Lambda(T)=3 n k_{B} v\left(\frac{T}{\Theta_{D}}\right)^{3 \Theta_{D} / T} \int_{0}^{\Theta^{4}} l(x) \frac{x^{4} \mathrm{e}^{x}}{\left(\mathrm{e}^{x}-1\right)^{2}} d x
$$

where $\Theta_{D}=v\left(h / k_{B}\right)\left(6 \pi^{2} n\right)^{1 / 3}, n$ is the number of atoms (molecules) per unit volume, $v$ is polarization - averaged sound velocity, $h$ is Planck constant $l(x)$ is phonon mean-free path. For $T \geq \Theta_{D}$ the phonon mean-free path can be found as

$$
l(x)=\frac{1}{C T} \frac{h^{2} v^{2}}{k_{B}^{2} T^{2} x^{2}},
$$

where $C$ is a coefficient. Since the mean-free path of the phonons cannot be smaller than the half wavelength $l(x)=$ $=a \lambda / 2$, where $a \approx 1$, the "diffusivity edge" $\Theta_{*}$ is

$$
\Theta_{*}=2 h v / a k_{B} C T
$$

The modes whose mean-free path is $a \lambda / 2$ are denoted as "diffuse" modes. It is assumed, that $\Theta_{*} \leq \Theta_{D}$ otherwise $\Theta_{*}=\Theta_{D}$. The thermal conductivity integral can be subdivided into two parts describing the contributions to thermal conductivity from low-frequency phonons $\Lambda_{\mathrm{ph}}$ and high-frequency "diffuse" modes $\Lambda_{\text {dif: }}$

$$
\Lambda=\Lambda_{\mathrm{ph}}+\Lambda_{\mathrm{dif}}
$$

$$
\begin{gathered}
\Lambda_{\mathrm{ph}}(T)=3 n k_{B} v\left(\frac{T}{\Theta_{D}}\right)^{3} \cdot\left[\int_{0}^{\Theta * / T} l(x) \cdot \frac{x^{4} \mathrm{e}^{x}}{\left(\mathrm{e}^{x}-1\right)^{2}} d x\right] \\
\Lambda_{\text {dif }}(T)=3 n k_{B} v\left(\frac{T}{\Theta_{D}}\right)^{3} \cdot\left[\int_{\Theta_{*} / T}^{\Theta_{D} / T} \alpha \frac{v h}{2 k_{B} x T} \cdot \frac{x^{4} \mathrm{e}^{x}}{\left(\mathrm{e}^{x}-1\right)^{2}} d x\right]
\end{gathered}
$$

The lower limit to thermal conductivity $\Lambda_{\min }$ is reached if all vibrating modes have "diffusive" character: $l(\lambda)=a \lambda / 2$ and it can be written as

$$
\Lambda_{\min }=3 \alpha\left(\frac{\pi}{6}\right)^{1 / 3} n^{2 / 3} k_{B} v\left(\frac{T}{\Theta_{D}}\right)^{2} \int_{0}^{\Theta_{D} / T} \frac{x^{3} \mathrm{e}^{x}}{\left(\mathrm{e}^{x}-1\right)^{2}} d x
$$

The least-square fitting to the smoothed thermal conductivity was performed for the highest-density sample with $V_{m}=95.6 \mathrm{~cm}^{3} /$ mole. Unfortunately, no experimental data on the sound velocity in solid $n$-hexane are available presently. We therefore used value $v=2.4 \times 10^{3} \mathrm{~m} / \mathrm{s}$ for liquid $n$-hexane immediately after melting [17] and varied the parameters $\mathrm{C}$ and $\alpha$. The best agreement with the experimental was achieved with $\mathrm{C}=6.810^{-10} \mathrm{~cm} / \mathrm{K}$ and $\alpha=$ $=1.86$. These coefficients $\mathrm{C}$ and $\alpha$ are closed to those of ethane and propane (see Table 3).

Table 3 . The average sound velocity $v$, and the fitting parameters $\mathrm{C}$ and $\alpha$.

\begin{tabular}{c|c|c|c}
\hline \hline Substance & $v \times 10^{-5} \mathrm{~cm} / \mathrm{s}$ & $C \times 10^{9} \mathrm{~cm} / \mathrm{K}$ & $\alpha$ \\
\hline \hline $\mathrm{C}_{2} \mathrm{H}_{6}$ & 1.42 & 1.06 & 1.84 \\
$\mathrm{C}_{3} \mathrm{H}_{8}$ & 1.95 & 1.1 & 2.64 \\
$\mathrm{C}_{6} \mathrm{H}_{14}$ & 2.4 & 0.68 & 1.86 \\
\hline \hline
\end{tabular}


The smoothed values of thermal conductivity (dark squares), the fitting curve (solid line) and the contributions of low-frequency phonons $\Lambda_{\mathrm{ph}}$ and diffuse modes $\Lambda_{\text {dif }}$ (dashed lines) are shown in Fig. 3. Here as dot-dashed line is also shown the lower limit to thermal conductivity, calculated according to Eg. (7). It is seen that the "diffuse" behavior of the vibrational modes becomes evident at $T>130 \mathrm{~K}$. Up to $T=200 \mathrm{~K}$ the contribution of "diffuse" modes is smaller than of phonons, which agrees well with the large jump of thermal conductivity observed on melting.

\section{Conclusion}

The isochoric thermal conductivity of solid n-hexane $\mathrm{C}_{6} \mathrm{H}_{14}$ has been investigated on three samples of different density in the temperature interval from $100 \mathrm{~K}$ to the onset of melting. In all the cases the isochoric thermal conductivity varied following a dependence which is weaker than $\Lambda \propto 1 / T$. The isobaric thermal conductivity exhibits closely similar behavior in short and long-chain $n$-alkanes. On the transition from the ordered phase to a liquid the thermal conductivity of the $n$-alkanes starting with propane changes nearly twice and is independent of the total entropy of transitions and the chain length. Such change is much smaller in the case of spherical and elliptic molecules. This can be related to the higher degree of orientational ordering in solid $n$-alkanes as compared to spherical molecules. The contributions of low-frequency phonons $\Lambda_{\mathrm{ph}}$ and "diffuse modes" $\Lambda_{\text {dif }}$ to the thermal conductivity are calculated. Unlike crystals consisting of the globular-shape molecules $\Lambda_{\text {ph }}$ is smaller than $\Lambda_{\text {dif }}$, which agrees well with the large jump of thermal conductivity observed on melting.

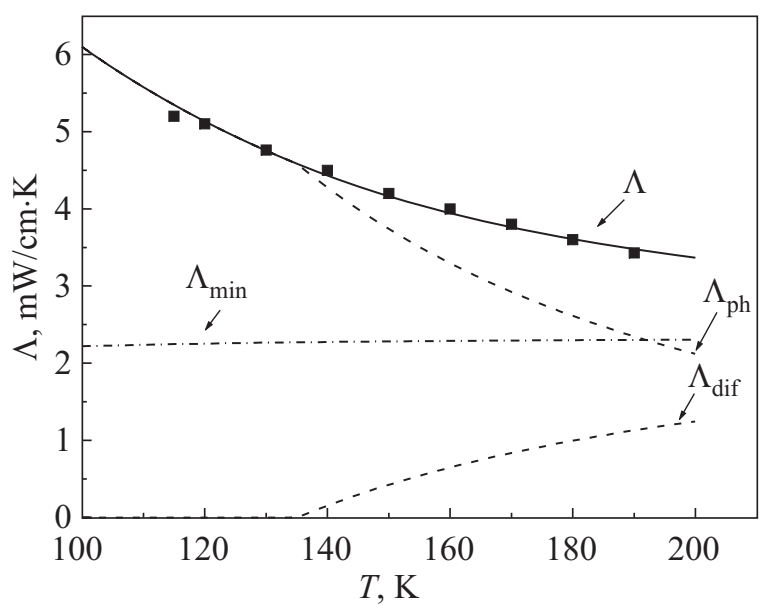

Fig. 3. The curve fitted to the smoothed experimental thermal conductivity of sample №1 and the calculated contributions to thermal conductivity from low-frequency phonons $\Lambda_{\text {ph }}$ and "diffuse" modes $\Lambda_{\text {dif. }}$ The dash-and-dot line is for the lower limit of the lattice thermal conductivity $\Lambda_{\text {min }}$.
1. A.I. Kitaigoroddkiy, Molecular Crystals (in Russian), Nauka, Moscow (1971).

2. R. Boese, H. C. Weiss, and D. Blaser, Angew. Chem. Int. Ed. 38, 988 (1999).

3. H.G. Olf and B. Fanconi, J. Chem. Phys. 59, 534 (1973).

4. A. Marbeuf and R. Brown, J. Chem. Phys. 124, 054901 (2006).

5. H. Forsman and P. Andersson, J. Chem Phys. 80, 2804 (1984).

6. V.A. Konstantinov, V.G. Manzhelii, V.P. Revyakin, and S.A. Smirnov, Physica B262, 421 (1999).

7. V.A. Konstantinov, V.P. Revyakin, and V.V. Sagan, Fiz. Nizk. Temp. 32, 905 (2006) [Low Temp. Phys. 32, 689 (2006)].

8. V.A. Konstantinov, V.P. Revyakin, and V.V. Sagan, Fiz. Nizk. Temp. 35, 735 (2009) [Low Temp. Phys. 35, 577 (2009)].

9. D.R. Douslin and H.M. Huffman, J. Am. Chem. Soc. 68, 1704 (1946).

10. N. Norman and H. Mathisen, Acta. Chem. Scand. 15, 1755 (1961).

11. W.B. Nelligan, D.J. LePoire, T.O. Brun, and R. Kleb, J. Chem. Phys. 87, 2447 (1987).

12. L.G. Brunel and D.A. Dows, Spectrochim. Acta 30A, 929 (1974).

13. V.A. Konstantinov, S.A. Smirnov, and V.P. Revyakin, Instr. Exp. Tech. 42, 133 (1999).

14. B.A. Younglove and J.F. Ely, J. Phys. Chem. Ref. Data, 16, 577 (1987).

15. D.G. Cahill, S.K. Watson, and R.O. Pohl, Phys. Rev. B46, 6131 (1992).

16. V.A. Konstantinov, Low Temp. Phys. 29, 567 (2003) [Fiz. Nizk. Temp. 29, 422 (2003)].

17. V.K. Sachdeva and V.S. Nanda, J. Chem Phys. 75, 4745 (1981). 